\title{
Modeling pathological intracranial pressure waveforms in idiopathic intracranial hypertension
}

\author{
S. A. Stevens ${ }^{1}$, W. D. Lakin ${ }^{2}$, N. J. Thakore ${ }^{3}$, P. L. Penar ${ }^{2}$ \\ \& J. Stimpson ${ }^{1}$ \\ ${ }^{1}$ Champlain College, USA \\ ${ }^{2}$ The University of Vermont, USA \\ ${ }^{3}$ MetroHealth Medical Center and Case Western Reserve University, USA
}

\begin{abstract}
Idiopathic intracranial hypertension (IIH) is a syndrome of unknown cause characterized by elevated intracranial pressure (ICP). By representing an abnormally collapsible sinus with a downstream Starling-like resistor, our previous modeling efforts have produced explanations for various phenomenon associated with IIH. Recent reports indicate that many patients with chronic daily headaches have been found to actually be suffering from IIH without papilledema (IIWOP). Moreover, these patients often demonstrate hypertensive B-waves and plateau-like waves upon continuous ICP monitoring. Our previous results did not produce such pathological wave-forms. The current model, described here, resolves this deficit by representing a semi-collapsible sinus by a refined resistor based on experimental data. With this revision the model exhibits low frequency, high amplitude ICP waves in milder cases of sinus collapsibility. As collapsibility increases further, so does the duration of the waves until they are replaced by two stable states, one of normal pressures and one of elevated pressures. In this domain the model behaves in much the same way as our previous work. Thus, the current model reproduces the results of previous versions and additionally exhibits the high amplitude spikes and wave-forms seen clinically in milder cases of IIH.
\end{abstract}




\section{Introduction}

Idiopathic intracranial hypertension (IIH), is a syndrome of unknown cause characterized by elevated intracranial pressure (ICP). It presents with symptoms of headache, nausea, vomiting, papilledema, and visual obscurations. A stenosis or tapering of the transverse sinuses has been observed in many patients suffering from IIH [1]. The role played by this stenosis in the etiology of IIH has been a matter of some dispute.

There are also indications that $\mathrm{IIH}$, in a subclinical form without papilledema (IIHWOP), may be more common than previously realized. A study by Bono et al. [2] revealed that $6.7 \%$ of 724 migraine patients had bilateral transverse sinus stenosis and $67.8 \%$ of these were diagnosed with IIHWOP. A study by Torbey et al. [3] also suggests that many patients with chronic daily headache (CDH) may actually be suffering from IIHWOP. Furthermore, of the ten patients examined in the Torbey study, all ten exhibited hypertensive B-waves and nine of the ten exhibited plateau or 'near-plateau' waves during continuous CSF pressure monitoring.

Recently, we reported [4,5] the results of modeling studies that suggest the observed sinus stenosis and persistent hypertension of IIH may be a physiological manifestation of a stable steady-state of elevated pressures predicted by the studies to exist when the transverse sinus is sufficiently collapsible. These results provide explanations for the following phenomenon associated with IIH: 1) the observed sinus stenosis, 2) intermittent occurrence of symptoms, 3 ) large pressure gradients observed across the transverse sinus, 4) the long-term relief that has been observed following lumbar puncture, and 5) the possible persistence of sinus stenosis after the hypertension has been resolved via pharmaceutical treatment and weight loss. These simulations do not, however, predict the prevalence of hypertensive ICP spikes (B-waves) or plateaus observed by Torbey in IIHWOP patients.

The lumped-parameter model presented here is a refinement of the model developed by Stevens et al. [5]. This previous modeling effort introduced a 'downstream' Starling-like resistor at the location of the transverse sinus. The principal difference between the current model and the previous model is that the description of the down-stream resistor has been modified to more closely reflect the data provided by Heil [6]. This resistor is placed at the location of the transverse sinuses in the model where the stenosis has been observed clinically.

\section{The model}

The lumped-parameter model utilized in this study is depicted in Figure 1. Here, the intracranial region is divided into four compartments. Compartment $C$ consists of the intracranial arteries and capillaries. This vasculature leads into the veins and saggital sinus which are represented by compartment $S$. Blood then drains through the transverse sinuses and into the jugular veins in compartment $V$. Brain tissue and cerebrospinal fluid (CSF) are lumped together to form compartment $F$. 


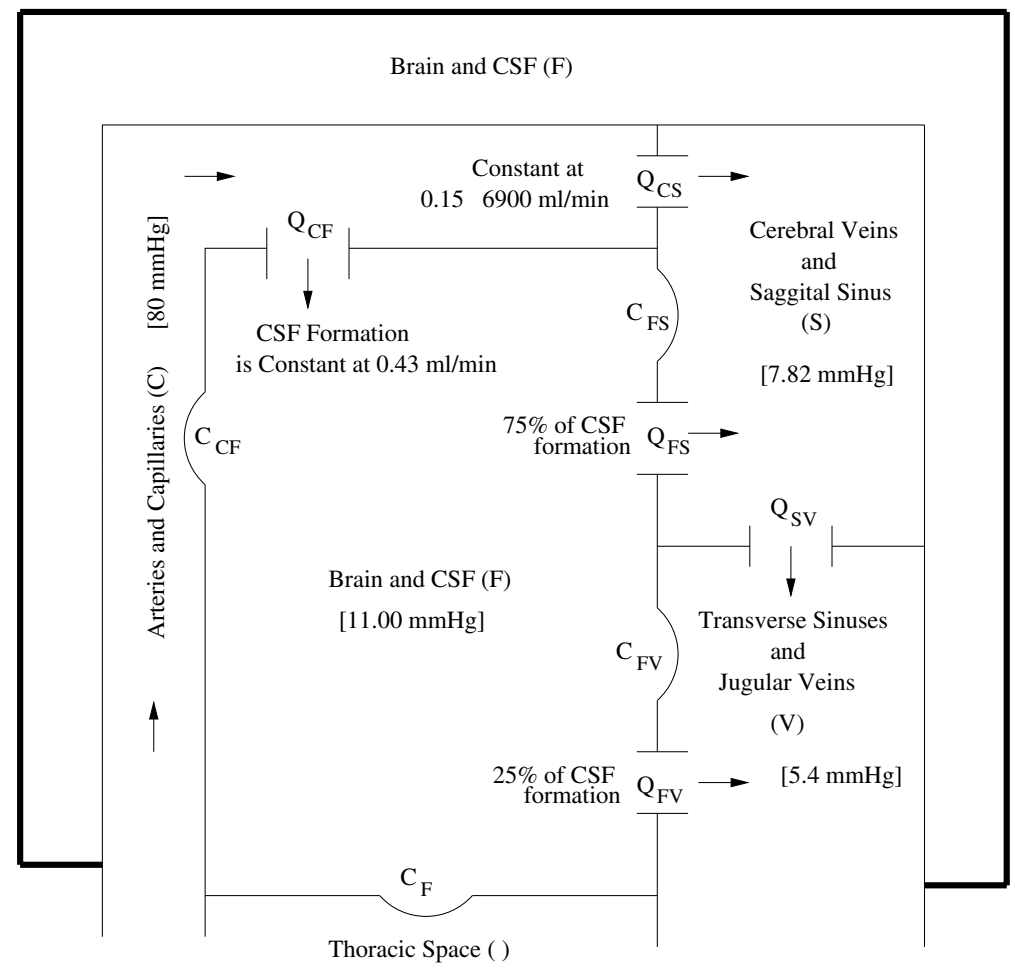

Figure 1: A diagram of the lumped-parameter model. Compartment labels are indicated in parentheses. $Q_{i j}$ represents fluid flow from compartment $i$ to compartment $j$ as determined by equation (1). Arrows indicate the customary direction of flow. The normal, base-value, pressure of each compartment is given in square brackets. Compliance terms $C_{i j}$ represent a deformable membrane between compartments $i$ and $j$ across which volume changes may be accommodated through equation (3). The pressures and flows depicted here are from Stevens et al. (2006) [5].

\subsection{Model assumptions}

The following basic assumptions lead to the fully time-dependent differential equations that govern the pressure dynamics of this system:

Assumption 1: All fluids are considered isothermal and incompressible.

Assumption 2: Cerebral blood flow $\left(Q_{C S}\right)$ and CSF production $\left(Q_{C F}\right)$ will be considered constant except in simulations of cerebral blood flow perturbations where $Q_{C S}$ is allowed to vary with time as a forcing term.

Assumption 3: All other flows $\left(Q_{F S}, Q_{F V}\right.$, and $\left.Q_{S V}\right)$ are related to pressure differences by the hydrodynamic version of Ohm's law; 


$$
Q_{i j}=\frac{P_{i}-P_{j}}{R_{i j}}=\frac{P_{i j}}{R_{i j}},
$$

where $Q_{i j}$ is the flow from compartment $i$ to compartment $j, P_{i}$ and $P_{j}$ are the spatially-averaged pressures of compartments $i$ and $j$, respectively, $P_{i j}=P_{i}-P_{j}$, and $R_{i j}$ is the lumped resistance.

Assumption 4: A partially-collapsible transverse sinus is represented by a downstream Starling-like resistor between compartments $\mathrm{S}$ and V. Similar resistors have been used previously to help explain various aspects of IIH [4, 5]. As in these previous works, the resistance term will be a decreasing function of the downstream transmural pressure. However, unlike previous versions, the representation here is linearly dependent on downstream transmural pressure and involves both a maximum and minimum resistance value. Specifically, this resistance function is defined by

$$
R_{S V}=\left\{\begin{array}{ccc}
p \bar{R}_{S V} & \text { if } & P_{F V}<P_{F V}^{\min } \\
{\left[1+m\left(P_{F V}-\bar{P}_{F V}\right)\right] \bar{R}_{S V}} & \text { if } & P_{F V}^{\min } \leq P_{F V} \leq P_{F V}^{\max } \\
q \bar{R}_{S V} & \text { if } & P_{F V}>P_{F V}^{\max }
\end{array} .\right.
$$

where

$$
P_{F V}^{\min }=\bar{P}_{F V}-\frac{1-p}{m} \quad \text { and } \quad P_{F V}^{\max }=\bar{P}_{F V}+\frac{q-1}{m} .
$$

Here, $\bar{P}_{F V}$ and $\bar{R}_{S V}$ represent the normal values of the pressure difference $P_{F V}$ and the resistance $R_{S V}$, respectively. The form of this resistor is based on the relationship between downstream transmural pressure and the pressure drop for constant fluid flow through a collapsible tube reported by Heil [6]. A graph of the present form of this resistor is depicted in Figure 2.

There are three relevant parameters in the present Starling-like resistance term. First, $m$ describes the initial collapsibility of the vessel. Second, $p$ defines the minimal resistance, $p \bar{R}_{S V}(0<p<1)$, when the vessel is in its maximally open state. Third, $q$ defines the maximal resistance, $q \bar{R}_{S V}(q \geq 1)$, when the vessel is in its maximally collapsed state. This maximum is imposed to accommodate alternative drainage routes and/or a geometry that may prevent complete collapse [8]. If the sinus is fully rigid, the parameters in (2) take the values $p=q=1$ and $m=0$. In this case, the resistance is constant at $\bar{R}_{S V}$.

Assumption 5: The deformation of the membrane between adjacent compartments is a function of the change in the pressure difference between these compartments. That is,

$$
\frac{d V_{i j}}{d t}=C_{i j} \frac{d}{d t}\left[P_{i}-P_{j}\right]=C_{i j} \dot{P}_{i j},
$$




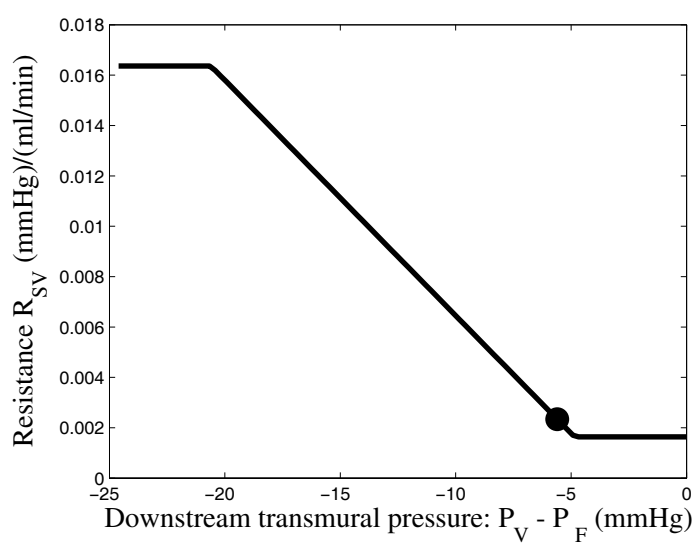

Figure 2: A graph of the resistance term $R_{S V}$ defined by equation (2) with $m=$ $.4, p=0.7$, and $q=7$. The large dot indicates the base-value state $\left(\bar{P}_{V F}, \bar{R}_{S V}\right)$.

where $V_{i j}$ denotes the volume of the cup formed at the interface of compartments $i$ and $j$, and $\dot{P}_{i j}$ represents the time derivative of the pressure difference $P_{i j}$. Here, $C_{i j}$ denotes the local compliance between the two compartments [9].

\subsection{Governing equations}

The model's governing differential equations are obtained by imposing conservation of mass in each compartment. As the fluid is considered incompressible, this requirement is equivalent to a conservation of volume. Imposing this constraint in compartments $F$ and $S$ results in the following equations

$$
\begin{aligned}
& Q_{C F}-Q_{F S}-Q_{F V}=\frac{d V_{F}}{d t}, \\
& Q_{C S}+Q_{F S}-Q_{S V}=\frac{d V_{S}}{d t} .
\end{aligned}
$$

Here, the right hand sides denote the change in volume with respect to time. Applying Assumptions 2, 3, and 5, the model's governing equations now become

$$
\begin{gathered}
\bar{Q}_{C F}-\frac{P_{F S}}{R_{F S}}-\frac{P_{F V}}{R_{F V}}=C_{F S} \dot{P}_{F S}+C_{F V} \dot{P}_{F V}+C_{C F} \dot{P}_{F C}+C_{F Y} \dot{P}_{F Y} \\
\bar{Q}_{C S}+\frac{P_{F S}}{R_{F S}}-\frac{P_{S V}}{R_{S V}}=C_{F S} \dot{P}_{S F} .
\end{gathered}
$$

For the sake of restricting the scope of our analysis to the relationship between $P_{F}$ and $P_{S}$, the pressures $P_{Y}, P_{C}$, and $P_{V}$ will all be held constant at their prescribed base-values. 


\subsection{The steady-state equations}

The first step in a steady-state analysis of the current model is to set the time derivative terms in the governing equations (6) and (7) to zero. The resulting system of algebraic equations for the steady-state now becomes

$$
\begin{aligned}
& f\left(P_{F}, P_{S}\right)=\bar{Q}_{C F}-\frac{P_{F S}}{R_{F S}}-\frac{P_{F V}}{R_{F V}}=0 \\
& g\left(P_{F}, P_{S}\right)=\bar{Q}_{C S}+\frac{P_{F S}}{R_{F S}}-\frac{P_{S V}}{R_{S V}}=0 .
\end{aligned}
$$

respectively.

In the parameter identification process, the resistance terms are calculated in such a way that the base-value pressures $P_{F}=\bar{P}_{F}$ and $P_{S}=\bar{P}_{S}$ will satisfy the steady-state equations.

\subsection{Stability analysis}

The fully time dependent system of differential equations (6) and (7) can be put into matrix form as

$$
\left(\begin{array}{cc}
C_{F S}+C_{F Y}+C_{C F}+C_{F V} & -C_{F S} \\
-C_{F S} & C_{F S}
\end{array}\right)\left(\begin{array}{c}
\dot{P}_{F} \\
\dot{P}_{S}
\end{array}\right)=\left(\begin{array}{c}
f\left(P_{F}, P_{S}\right) \\
g\left(P_{F}, P_{S}\right)
\end{array}\right) .
$$

Here, the functions $f$ and $g$ are defined in the steady-state equations (8) and (9). The stability properties of steady-state solutions can now be determined from the eigenvalues of the Jacobian matrix for the above system.

\section{Results}

The steady-state results presented here are based on explicitly solving equations (8) and (9) for all steady-state solution pairs $\left(P_{F}, P_{S}\right)$, and time dependent simulations were performed by numerically integrating equation (10).

\subsection{Steady-state solutions and stability}

Figure 3 depicts the bifurcation diagram for the system (10) in terms of the CSF pressure $P_{F}$. As seen here, the value of $m$ determines the stability of the base-value state $\left(\bar{P}_{F}, \bar{P}_{S}\right)$ and whether there are additional steady-states present. In terms of the steady-state solution $P_{F}$ there is a unique stable steady state $P_{F}=\bar{P}_{F}$ for $m<m_{2}$. Consequently, for values of $m$ less than $m_{2}$, there is only the basevalue state, and it is globally stable. A Hopf bifurcation occurs at $m_{2}$ resulting in a stable limit cycle of pressures. The pulse pressure (max - min) of these cycles increases quickly with $m$ to a limiting value of $P_{H}-P_{L}$. At $m_{L}$ a saddle-node bifurcation occurs producing a stable hypotensive state. Soon after, at $m_{H}$, a stable 


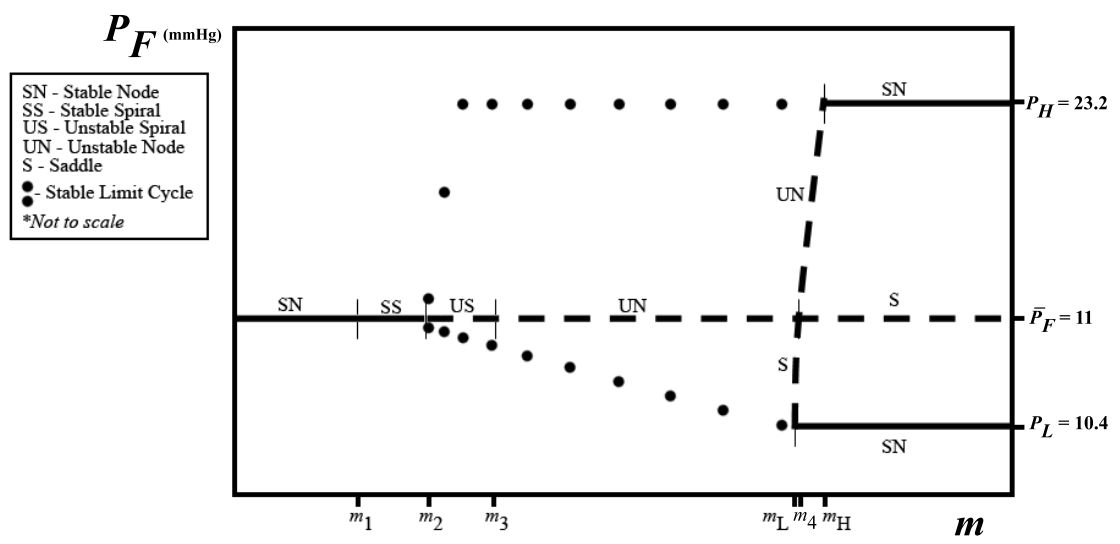

Figure 3: A bifurcation diagram of the steady-state solution $P_{F}$ with respect to the rigidity parameter $m$ for $p=0.7$ and $q=7$. When $m<m_{2}$, the horizontal line at $P_{F}=\bar{P}_{F}$ represents the unique, globally-stable, base-value state. A Hopf bifurcation occurs at $m_{2}$ resulting in a stable limit cycle of ICP's. The pulse pressure of these cycles increases quickly to the limiting value of $P_{H}-P_{L} \approx P_{H}-\bar{P}_{F}$. At $m_{L}$ a saddle-node bifurcation occurs producing a stable hypotensive state. Soon after, at $m_{H}$, a nontraditional bifurcation occurs and a stable hypertensive state is introduced. The level of the hypotensive state $\left(P_{L}\right)$ and the stable hypertensive state $\left(P_{H}\right)$ are determined by the rigidity parameters $p$ and $q$ respectively.

hypertensive state is introduced. For $m>m_{H}$ the stable hypotensive and hypertensive states persist. At this stage the current model reflects the same behavior that is described by Stevens et al. [5]. As such, the focus of this investigation and time-dependent simulations will be in the parameter domain where self-excited oscillations are present $\left(m_{2}<m<m_{L}\right)$.

\subsection{Low frequency self-excited waves - limit cycles}

When the rigidity parameter $m$ falls between $m_{2}$ and $m_{L}$ there are no stable steady-state solutions but rather stable limit cycles of relatively low frequency pressure oscillations. Figure 4 depicts several examples of these self-excited oscillations. These pulsations progress from high-frequency spike-like waves when $m$ first crosses $m_{2}$ (panels A and B), to less frequent plateau-like waves of increasing period (panels $\mathrm{C}$ and $\mathrm{D}$ ).

\subsection{Cerebral blood flow disturbances}

When the rigidity parameter $m$ falls between $m_{2}$ and $m_{L}$ the system is very sensitive to pressure and flow disturbances that may naturally occur during normal 
Panel A: $m=0.445$

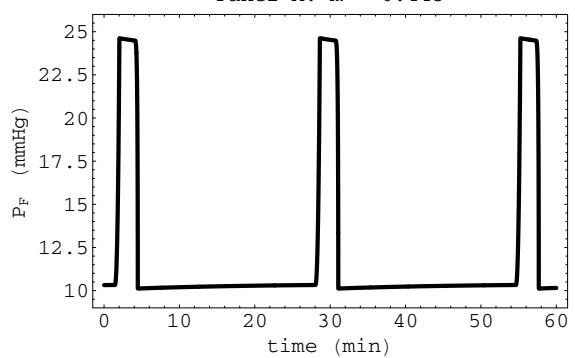

Panel $\mathrm{C}: \mathrm{m}=0.46$

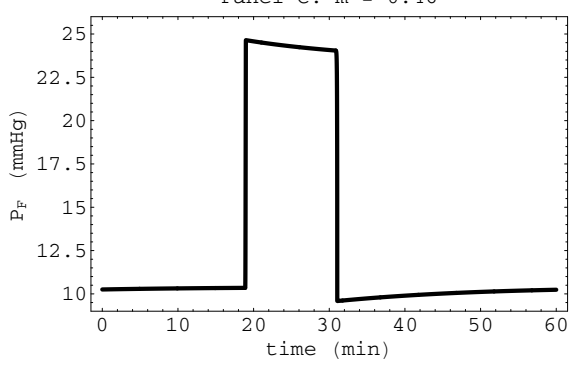

Panel B: $m=0.45$

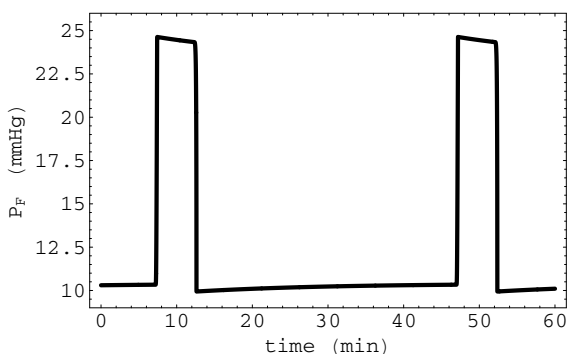

Panel D: $m=0.47$

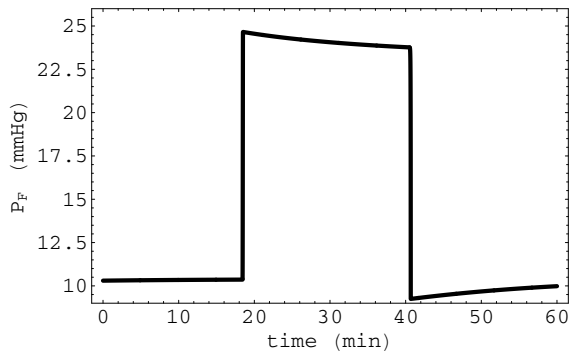

Figure 4: This sequence of simulations demonstrates that as $m$ increases from 0.445 (upper left) to 0.47 (lower right) the self-excited wave frequency diminishes and the plateau period increases. The first graph demonstrates waves that are similar to clinically observed hypertensive B-waves and the later graphs are similar in wave-length and amplitude to clinically observed A-waves. In all four cases, the rigidity parameters $p$ and $q$ were given numerical values of 0.7 , and 7 respectively.

physiology. This is simulated by introducing a cerebral blood flow (CBF) 'spike' into the model via the flow term $Q_{C S}$. The duration of this spike is 6 seconds and it's magnitude is $10 \%$ of the normal level of CBF.

In simulations of normal physiology, where the sinuses are rigid and $m=0$, the CBF spike described above produces approximately a $0.2 \mathrm{mmHg}$ spike in ICP, a response that would go unnoticed physiologically and clinically. Conversely, in simulations of pathophysiology where the sinus is semi-collapsible with $m_{2}<$ $m<m_{L}$, this small CBF disturbance can cause a much more drastic ICP spike or a premature transition to a plateau wave. These responses are depicted in Figure 5 with $m=0.45$. The low frequency plateau waves are caused by the semicollapsible sinus (see panel B in Figure 4). When a mild CBF spike occurs shortly after a completed wave, a single hypertensive ICP spike results. When this same stimulus occurs closer to the beginning of the next plateau wave, it causes a plateau wave to occur sooner than it otherwise would. Therefore, when $m_{2}<m<m_{L}$, it is quite likely that hypertensive B-waves and plateau-like waves that could be 

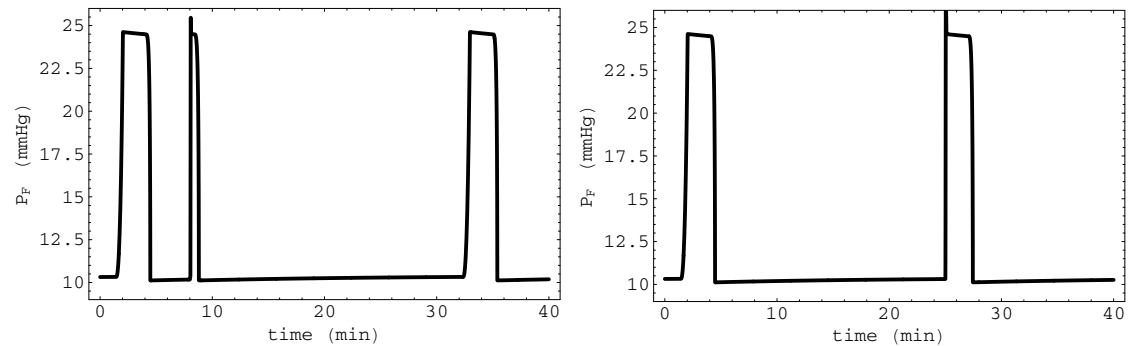

Figure 5: A six second $10 \%$ increase in cerebral blood flow $\left(Q_{C S}\right)$ initiates a hypertensive B-wave (left) when introduced shortly after a plateau wave and initiates an early plateau wave (right) when introduced later in the cycle. Here, $p=0.7, q=7$, and $m=0.445$ as in panel A of figure 4 .

missed during a single lumbar pressure measurement would be observed during long term ICP monitoring.

\subsection{Two stable states: hypotensive and hypertensive}

The saddle-node bifurcation that occurs at $m_{L}$ initially introduces two hypotensive states, and the unstable state quickly transitions to a stable hypertensive state. Here, small transient perturbations can cause permanent transitions between the two states. It should be noted that the magnitude of the hypertensive state is determined by the parameter $q$ and the hypotensive state is determined by the parameter $p$. Additionally, the hypotensive state is close enough to normal pressure that it would not be clinically recognized as hypotensive. In this parameter domain, the current model reflects the same behavior depicted by our previous model [5] and as such will not be elaborated upon further here.

\section{Conclusions}

The present refinement in the way a compressible transverse sinus is represented in a mathematical model of lumped-parameter type expands our previous ability to explain many of the various phenomenon associated with IIH. These included sinus stenosis, intermittent occurrence of symptoms, large pressure gradients across the transverse sinuses, and long-term relief after a single CSF withdrawal. In addition, the current model provides new insight into the existence of low-frequency, high-amplitude ICP waveforms observed in chronic daily headache patients diagnosed with IIHWOP.

\section{References}

[1] King, J., Mitchell, P., Thomson, K. \& Tress, B., Manometry combined with cervical puncture in idiopathic intracranial hypertension. Neurology, 58, pp. 26-30, 2002. 
[2] Bono, F., Messina, D., Giliberto, C., Critiano, D., Broussard, G., Fera, F., Condino, F., Lavano, A. \& Quattrone, A., Bilateral transverse sinus stenosis predicts iih without papilledema in patients with migraine. Neurology, 67, pp. 419-423, 2006.

[3] Torbey, M., Geocadin, R., Razumovsky, A., Rigamonti, D. \& Williams, M., Utility of csf pressure monitering to identify idiopathic intracranial hypertension without papilledema in patients with chronic daily headache. Cephalalgia, 24, pp. 495-502, 2004.

[4] Stevens, S., Lakin, W., Thakore, N., Penar, P. \& Tranmer, B., A modeling study of idiopathic intracranial hypertension. Modelling in Medicine and Biology VI, eds. M. Ursino et al., WIT-Press: South Hampton, UK, 2005.

[5] Stevens, S., Previte, M., Lakin, W., Thakore, N., Penar, P. \& Hamschin, B., Idiopathic intracranial hypertension and transverse sinus stenosis: A modeling study. Math Med Biol, 24(1), pp. 85-109, 2006.

[6] Heil, M., Stokes flow in collapsible tubes: computation and experiment. $J$ Fluid Mech, 353, pp. 285-312, 1997.

[7] Stevens, S., Lakin, W. \& Penar, P., Modeling steady-state intracranial pressures in supine, head-down tilt, and microgravity conditions. Aviat Space Environ Med, 76, pp. 329-338, 2005.

[8] De Simone, R., Marano, E., Fiorillo, C., Briganti, F., Di Salle, F., Volpe, A. \& Bonavita, V., Sudden re-opening of collapsed transverse sinuses and longstanding clinical remission after a single lumbar puncture in a case of idiopathic intracranial hypertension. pathogenetic implications. Neurol Sci, 25(6), pp. 342-344, 2005.

[9] Stevens, S. \& Lakin, W., Local compliance effects on the global CSF pressure-volume relationship in models of intracranial pressure dynamics. Mathematical and Computer Modelling of Dynamical Systems, 6(4), pp. 445-465, 2001. 